\title{
Frequency of replication errors in colorectal cancer and their association with family history
}

\author{
S R Brown, P J Finan, L Cawkwell, P Quirke, D T Bishop
}

\begin{abstract}
Background-Replication errors (RERs) characterise tumours of hereditary nonpolyposis colorectal cancer (HNPCC). RER status may therefore improve identification of such families previously diagnosed by family history alone.

Aims-To assess RER and HNPCC frequency within a population of colorectal cancer patients and a regional population of family history defined (Amsterdam criteria) HNPCC families.

Methods-Family history was assessed by personal interview in a population of 479 patients with colorectal cancer attending one follow up clinic. Seven fluorescently labelled microsatellites were used to investigate RER frequency in colorectal cancers from 89 patients of this population with varying degrees of family history and 20 Amsterdam criteria positive families (four with a known germline mutation, 16 with unknown mutation status) from the regional population.

Results-Only four of the follow up population $(0.8 \%)$ came from families meeting the Amsterdam criteria with only one showing RERs. The frequency of RERs was similar in the early onset cancer group (less than 50 years of age), those with a family history, and those with no family history of colorectal cancer. From the regional population, RERs were identified in $4 / 4$ families with a mutation but only $8 / 16$ families with unknown mutation status.

Conclusions-No correlation was seen between RER status and strength of family history except in HNPCC families. Results also indicate that half of the Amsterdam criteria defined families do not exhibit RERs, perhaps suggesting a different mechanism of tumorigenesis.

(Gut 1998;43:553-557)
\end{abstract}

Keywords: hereditary non-polyposis colorectal cancer; replication errors

Imperial Cancer Research Fund, Genetic Epidemiology

Department, St James's University Hospital, Leeds, UK D T Bishop

Correspondence to: Mr S R Brown, Genetic Epidemiology Department, ICRF, St James's Hospital, Leeds LS9 7TF, UK.

Accepted for publication 3 April 1998 no obvious hereditary trait.

Of the main hereditary colorectal cancer syndromes, FAP is easily recognised clinically because of the characteristic phenotypic pres- entation of multiple colonic polyps. However, with HNPCC there is no obvious phenotypic marker of disease. One method of diagnosis relies on recognition of a strong family history, often of early onset and predominantly right sided colorectal cancer. ${ }^{4}$

In an effort to standardise reporting of putative HNPCC families, the Amsterdam criteria were proposed as the minimum basis for diagnosis. These state that for a family to be considered to have HNPCC, after familial adenomatous polyposis is excluded, there should be at least three affected relatives with colorectal cancer (one a first degree relative of the other two) with at least one early onset cancer (less than 50 years of age), at least two generations affected, and a pedigree that suggests an autosomal dominant inheritance. ${ }^{5}$

There are problems with using these criteria as a basis for diagnosis of any hereditary syndrome. Under diagnosis may occur because of the difficulty in obtaining and verifying a family history. Conversely, over diagnosis may occur because of chance clustering of a common disorder as well as shared environmental risk factors such as diet. ${ }^{6}$

Recently, inherited mutations in the mismatch repair genes have been implicated in the causation of HNPCC..$^{7-9}$ Mutation screening of these genes allows another method of identifying true HNPCC families. Unfortunately, because of the number and the large size of the mismatch repair genes and the absence of any mutation "hot spots", screening remains labour intensive and costly and is impracticable for a non-selected population. ${ }^{10}$

A possible screening alternative is to search for the phenotypic result of a mismatch repair gene mutation. Faulty repair results in accumulation of defects within tumour DNA during cell division, leading to replication errors (RERs), especially within microsatellite repeat markers. ${ }^{11}{ }^{12}$ Previous reports examining HNPCC families have suggested that this RER phenotype, recognised by novel alleles in tumour DNA not present in normal DNA, occurs in over $90 \%$ of HNPCC tumours. This is compared with only $10-15 \%$ of apparently sporadic tumours. ${ }^{12}{ }^{13}$ Therefore, even accounting for the relative preponderance of sporadic cancer, if two or more tumours from the same family are RER positive, then that family is more likely to represent a true HNPCC family. This provides a further method of HNPCC diagnosis.

We hypothesised that RER occurrence would correlate with the strength of family history of bowel cancer. If true, a knowledge of RER status might enhance the identification of 
families with a germline mismatch repair gene mutation. Using a large cohort of colorectal cancer patients attending one surgeon's practice, we assessed this relation. In addition, to supplement the number of families with strong family histories, we assessed the incidence of RER in a second population of Amsterdam criteria defined HNPCC families attending one regional genetics department.

\section{Materials and methods}

PATIENTS

Information was gathered on patients referred for resection of a colorectal malignancy to one consultant surgeon over a period of 10 years (between January 1987 and December 1996). Over this period detailed family histories have been obtained by interviewing patients or their surviving next of kin concerning dates and causes of death and colorectal cancer diagnoses of all known relatives. Attempts were made to verify all suspected diagnoses of cancer and uncertain causes of death by consulting cancer registries, histopathology reports, death certificates, and medical records. Patients with incomplete or unverified histories as well as those with inflammatory bowel disease were excluded from subsequent analysis. In addition, one patient whose colorectal cancer had been diagnosed through screening because of a family history of cancer, was excluded.

Familial aggregation of colorectal cancer was documented according to the number of patients in each group with none, one, or two affected first degree relatives. In addition, the incidence of FAP was documented along with those considered to belong to HNPCC families according to the Amsterdam criteria. Finally those patients with no family history who also had early onset cancers (less than 50 years of age) were included as a separate group.

A second cohort consisted of all patients from families meeting the Amsterdam criteria that had been referred to either the Yorkshire Regional Genetics Department or the Imperial Cancer Research Fund Genetic Epidemiology Department. Four families with a known mismatch repair gene defect were identified (an average of eight individuals with colorectal cancer per family) ${ }^{14}$ as well as 16 families who have not as yet undergone mutation analysis. All of these families were small; there was an average of 3.7 members with colorectal cancer per family.

For each of the Amsterdam criteria positive families from both populations, samples of normal and colorectal cancer paraffin wax embedded, formalin fixed material were obtained from at least two affected individuals. Similar samples were obtained from a representative proportion of the patients in the familial or early onset groups depending on the availability of material. In addition, age, sex, and tumour site matched samples were obtained from the group of patients with no affected relatives.

DNA EXTRACTION

For each sample a haematoxylin and eosin (H\&E) stained section was examined by a pathologist who marked areas of the highest tumour cell density as well as normal tissue. Subsequent sections were microdissected to obtain samples of normal and tumour cells. DNA was extracted using a Puregene extraction kit (Gentra Systems Inc., Minneapolis, USA). In four randomly selected tumours where no RERs were seen, DNA was reextracted using a different area of tumour. This was carried out because of the possibility of tumour RER heterogeneity and ensured that RER positive tumour cells were not being missed by inappropriate selection of tumour site.

\section{PRIMERS}

Seven microsatellite markers were used for each patient. Many of these markers have been used in previous RER studies. ${ }^{11}{ }^{13}$ Markers included the dinucleotide repeats D2S123, D2S391, D18S58, D3S1298, and D3S1611 as well as the mononucleotide repeats BAT25 and BAT40 and were all fluorescently labelled and purified as described previously. ${ }^{16}$ At least five markers were successfully analysed per tumour.

FLUORESCENCE POLYMERASE CHAIN REACTION Fluorescence polymerase chain reaction (PCR) was performed in $10 \mu \mathrm{l}$ aliquots using 1 $\mu 1$ of DNA (aprox. $0.2 \mathrm{ng} / \mu \mathrm{l}$ ) with $0.2 \mu \mathrm{l} 50 \times$ KlenTaq pDNA polymerase mix and $1 \mu 110 \times$ Klen Taq PCR reaction buffer (Clontech Laboratories Inc., California, USA), $200 \mu \mathrm{mol} / 1$ dNTPs, and $5 \mathrm{pmol} / 1$ of each primer. This was overlaid with mineral oil. The DNA was amplified in a thermal cycler (MJ Research Inc.) by one cycle at $95^{\circ} \mathrm{C}$ for 30 seconds followed by 33 cycles of both $61^{\circ} \mathrm{C}$ for 30 seconds and $95^{\circ} \mathrm{C}$ for 30 seconds with a final extension of $72^{\circ} \mathrm{C}$ for two minutes.

POLYACRYLAMIDE GEL ELECTROPHORESIS AND DATA ANALYSIS

The fluorescent PCR products were separated using $6 \%$ denaturing gels in a model 373A automated DNA sequencer (Applied Biosystems, California, USA) and subsequent analysis was carried out as previously described. ${ }^{16}$

\section{ASSESSMENT OF RER}

The RER phenotype was considered to be present if one or more consistent novel alleles were seen in tumour DNA compared with normal DNA (fig 1) in two or more markers. Cases where only one marker showed alterations were also documented.

STATISTICAL ANALYSIS

RER frequency in the familial and early onset groups was compared with the control group (no affected relatives) using contingency table analysis. Mean age for patients with RER positive and RER negative tumours from the Amsterdam criteria defined only families was compared using Student's $t$ test.

\section{Results}

FREQUENCY OF FAMILIAL CANCER

During the 10 year period, 661 patients have undergone surgery under one consultant. To 

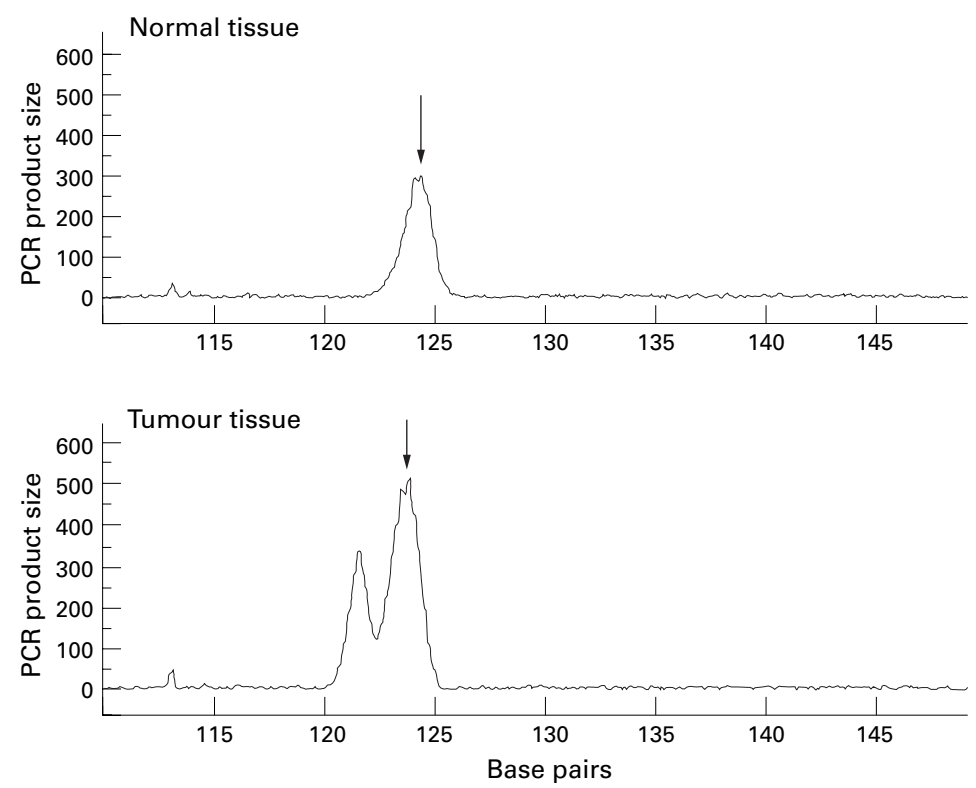

Figure 1 Microsatellite instability: a cross sectional image of the products of a polymerase chain reaction using one fluorescently labelled microsatellite marker. The upper panel represents normal tissue and illustrates one peak corresponding to the normal allele at this location (arrow). This is repeated in the bottom panel (arrow) but with a preceding novel allele.

Table 1 Results from analysis of family histories of patients from one consultant surgeon's practice

\begin{tabular}{lcc}
\hline & Number & $\%$ \\
\hline Full family history & 479 & - \\
Male & 261 & 54 \\
Female & 218 & 46 \\
Mean age (years) & 69 & - \\
$\quad$ Range & $28-94$ & - \\
Number of first degree relatives with colorectal cancer & 384 & 17 \\
$\quad$ None & 80 & 2.5 \\
One & 10 & 3 \\
Two & 15 & 0.2 \\
Patients with early onset cancer (<50 years), no family & 1 & 0.8 \\
$\quad$ history (mean age 43 years) & 4 & \\
Patients with FAP & & \\
Patients from families meeting the Amsterdam criteria & & \\
\hline
\end{tabular}

date 592 patients have been approached for a family history. Of these four were excluded because of inflammatory bowel disease and four because they were adopted. A further 56 patients had died and had no traceable next of kin while 34 patients and 15 next of kin refused to give a history. In the remaining 479 patients

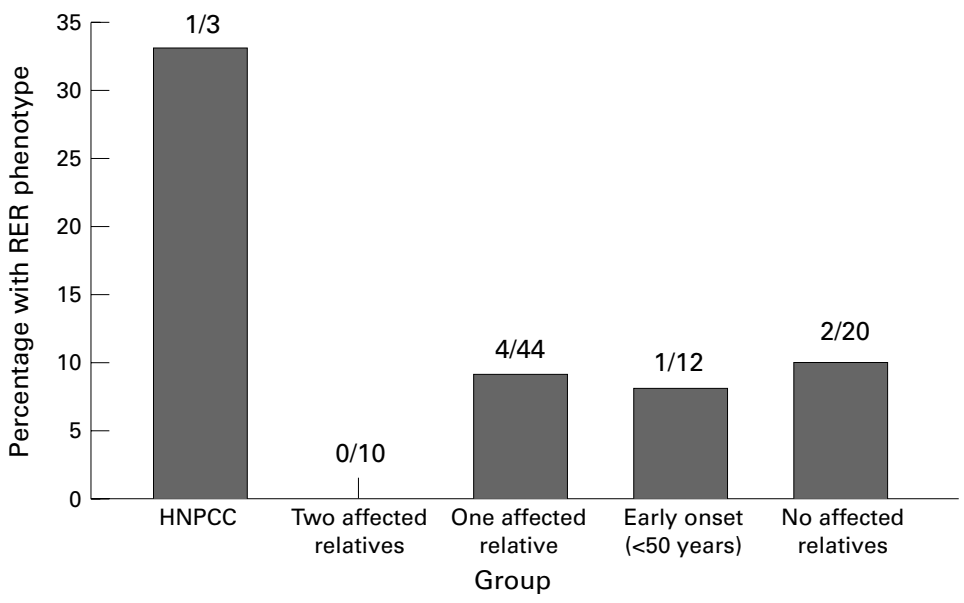

Figure 2 Comparison of the incidence of RER phenotype with the strength of family history for patients from one consultant surgeon's practice.

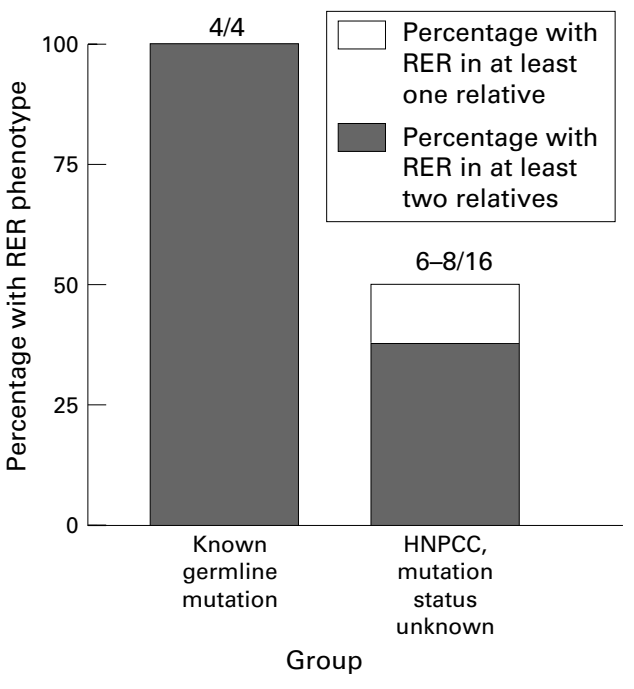

Figure 3 Incidence of RER phenotype for families with a known mismatch repair gene and those defined as HNPCC using family history only.

(73\% of the cohort) a detailed family history was obtained and verified.

Table 1 details familial aggregation for this cohort. Overall $20 \%$ of patients had some degree of family history. There was only one patient $(0.2 \%)$ with FAP and only four patients $(0.8 \%)$ from three families meeting the Amsterdam criteria.

RER STATUS-CORRELATION WITH FAMILY HISTORY

RER status was analysed in two affected individuals from the three families meeting the Amsterdam criteria and the RER phenotype was identified in only one family, being seen in both individuals. In addition the RER phenotype was seen in none of 10 patients $(0 \%)$ from the two first degree relative group, four of 44 $(9 \%)$ patients from the one first degree relative group, and one of $12(8 \%)$ patients from the early onset group. These frequencies were not statistically different to the group with no affected relatives where RER was detected in two of 20 patients (10\%) (see fig 2). RERs in all positive individuals occurred in two or more of the markers used.

RER STATUS IN FAMILY HISTORY DEFINED HNPCC FAMILIES

The RER phenotype was detected in at least two individuals from the four families with a known mismatch repair gene mutation. RERs occurred in more than two markers for each individual. However, in the smaller families (average 3.7 colorectal cancers per family) the RER phenotype was seen in two or more individuals in only six of the 16 families (fig 3). In two individuals from separate families DNA alterations were noted in only one of the seven markers. These individuals could not be described as having the RER phenotype by our strict definition. However, as both patients had a relative with RER, their families were regarded as possibly RER positive.

In these smaller families, no relation was seen between age or tumour site and the frequency of RER. The mean age for the RER 
positive patients was 57.5 years compared with 57.3 years for the RER negative cases $(t=0.01$, $\mathrm{df}=32, \mathrm{p}>0.5)$ and $44 \%$ of the RER positive tumours were right sided compared with $63 \%$ of the RER negative tumours $\left(\chi^{2}=2.04, \mathrm{df}=1\right.$, $\mathrm{p}>0.1$ ).

There were a total of 29 RER positive cases in our study with at least two markers showing instability. Overall, 76 of 173 (44\%) informative markers showed instability in these cases. There was no significant difference in this proportion of affected markers for the known mutation group (38\% of markers affected), the "Amsterdam criteria only" group ( $44 \%$ of markers affected), and the "sporadic" group ( $50 \%$ of markers affected).

Four randomly selected RER negative tumours were selected for re-extraction of the tumour DNA using a different area of tumour, but all remained negative.

\section{Discussion}

In the population of colorectal cancer patients studied, we observed a frequency of familial colorectal cancer of one in five patients, in agreement with several previous studies. ${ }^{1-3}$ However, the apparent frequency of hereditary disorders such as FAP and HNPCC was lower than several previous estimates. ${ }^{317} 18$ Only a very small proportion of patients from the early onset group as well as the familial groups had the RER phenotype, and could be considered as likely to be unrecognised HNPCC. Furthermore, none of the patients from families only just failing to meet the Amsterdam criteria, and therefore possibly most likely to be putative HNPCC, had the RER phenotype. This makes it unlikely that the frequency of HNPCC was underestimated using the Amsterdam criteria in the population studied. In fact, rather than underestimating HNPCC using family history this may have been overestimated. Analysis of RER in the three HNPCC families revealed only one family with the RER phenotype, implying that the other two families were not true HNPCC. It is interesting that a recent population study, again from the north of England, suggested a similar low HNPCC frequency of less than $1.7 \% .^{19}$

Coupled with these observations, we were also able to show no correlation with RER phenotype and strength of family history. This is consistent with previous studies ${ }^{20} 21$ and implies that, although some mechanism of genetic susceptibility probably exists in these patients, it is unlikely to involve mismatch repair defects.

A previous study on RER in early onset colorectal cancer patients showed that the RER phenotype is frequently observed in patients less than 35 years old at diagnosis. ${ }^{15}$ Although our numbers were small, our results were not consistent with this observation. However, it should be noted that almost all of our sporadic cancer patients were over 35 years old when they developed cancer.

Previous reports have also suggested that the frequency of the RER phenotype is as high as $92 \%$ of HNPCC families. ${ }^{12}{ }^{22} 23$ A number of these reports examined relatively small num- bers of patients and were heavily biased towards those of Scandinavian origin. As many of these cases are in fact caused by a very small number of mutations within hMLH1 spread through a common ancestor, it has been suggested that they may not be representative. ${ }^{23}$ A more recent study involving families from New Zealand, Europe, and North America, however, gave results consistent with the mainly Finnish studies. ${ }^{23}$

One criticism of all of these reports is the size of the families studied. Little information was given concerning size in the majority of the reports but the implication was that the families studied were large with numerous affected individuals. These families were not representative of the majority of family history defined families attending our surgical or genetic specialists.

In order to examine the incidence of the RER phenotype in small HNPCC families and because the number of such families was small in our clinic population, we examined regional genetic referrals. Analysis confirmed that RER was a feature of HNPCC families where there was a known mismatch repair gene mutation. Our numbers here were small but instability was nevertheless seen in all eight individuals studied. However, in smaller Amsterdam criteria positive families the incidence of instability was considerably less than previously reported. ${ }^{11}{ }^{12}{ }^{23}$ We estimate that a maximum of $50 \%$ of these small families have two or more individuals with the RER phenotype.

Possible explanations for these differing results relate to methodology. Because of tumour heterogeneity clones of cells may exist where there is no RER phenotype alongside RER positive clones. ${ }^{24}$ We sampled areas of tumour with the highest concentration of tumour cells and it may be that these areas contained RER negative clones while RER positive cells existed elsewhere, resulting in an underestimation of RER incidence. However, previous studies have incorporated similar methodology. ${ }^{23}$ Furthermore, repeat sampling of other tumour areas in four of our RER negative cases failed to show any difference.

Previous studies have incorporated a radioactive PCR method combined with autoradiography for detection of instability. ${ }^{12} 23 \mathrm{An}$ intrinsic problem with this method, particularly when using dinucleotide repeat markers, is the production of stutter bands, thought to be caused by the Taq polymerase in the PCR failing to read through the repeat region, thereby generating smaller fragments. ${ }^{25}$ These stutter bands can make autoradiographs difficult to interpret, particularly in identifying replication errors. Furthermore, lane to lane variation can be a problem particularly with the need to compare normal and tumour lanes. Both issues may lead to over identification of replication errors in studies incorporating similar techniques.

We used an automated DNA sequencer which can detect fluorescently labelled PCR products and allow rapid and improved assessment of replication errors with easier differentiation of stutter bands and, as a size standard 
is co-electrophoresed with every sample, lane to lane variation is not a significant issue.

It is possible that some RER positive tumours were missed because of insufficient microsatellite markers. Although replication errors are thought to be a widespread phenomenon, the sheer size of the genome and number of microsatellite regions means that a substantial instability phenotype may exist but not be detected with our seven microsatellites. However, both number and type of primers used were similar to those use in previous studies. ${ }^{12} 1523$ In fact, in the most recent of these studies, showing a $92 \%$ incidence of HNPCC families with the RER phenotype, ${ }^{23}$ the primers used were almost identical to this study with the only difference being the use of only four markers compared with at least five, and usually seven, informative markers in our study. In addition, in the group of patients with a known mismatch repair gene mutation who would be expected to have the RER phenotype, all showed alterations in at least two of the seven microsatellites used.

The most likely explanation for the result disparity is family size with an average of only four affected individuals in each of our families. The lower frequency of RER in these smaller families suggests that some may not be genuine examples of HNPCC and that the criteria are insufficiently stringent in such cases resulting in overdiagnosis of the syndrome. Jass et $a l^{26}$ noted a similar reduced frequency of the RER phenotype in small HNPCC families (mean number of affected individuals 3.4) compared with larger families (mean number of affected individuals 10.1).

Our results have confirmed many previous observations concerning the frequency of familial cancer as well as the absence of correlation with RER and family history. However, we also suggest that HNPCC, defined by the Amsterdam criteria combined with the RER phenotype, forms a very small part of the cancer burden in a typical surgical practice. The observed frequency of the RER phenotype in our regional genetic department referrals also suggests that only about $50 \%$ of Amsterdam criteria defined families are true HNPCC in that there is no mismatch repair gene defect to explain tumorigenesis. This result is in agreement with a recent publication investigating mismatch repair gene mutations in Amsterdam criteria positive kindreds as well as those where the criteria were not fully satisfied. ${ }^{27}$ Mutations were identified in $49 \%$ of these Amsterdam criteria positive families compared with only $8 \%$ of the criteria negative families. These authors also showed microsatellite instability tended to be a feature of the Amsterdam criteria positive families.
This work was supported by the Imperial Cancer Research Fund and the Yorkshire Cancer Research Campaign. It was presented at the Association of Coloproctologists of Great Britain and Ireland, Edinburgh, 1997.

1 Lovett E. Family studies in cancer of the colon and rectum. Br f Surg 1976;63:13-18.

2 Ponz de Leon M, Sassatelli R, Sacchetti C, et al. Familial aggregation of tumours in the three-year experience of a population based colorectal cancer registry. Cancer Res 1989;49:4344-8.

3 Stephenson B, Finan P, Gasgoyne J, et al. Frequency of familial colorectal cancer. Br f Surg 1991;78:1162-6.

4 Lynch H, Smyrk T. Hereditary nonpolyposis colorectal cancer. An updated review. Cancer 1996;78:1149-67.

5 Vasen HFA, Mecklin J-P, Meerakhan P, et al. The international collaborative group on hereditary nonpolyposis colorectal cancer. Dis Colon Rectum 1991;34:424-5.

6 Steinmetz K, Potter J. Colon cancer: a review of the epidemiology. Epidemiol Rev 1991;15:499-545.

7 Peltomaki P, Aaltonen LA, Sistonen P, et al. Genetic mapping of a locus predisposing to human colorectal canmapping of a locus predisposing 1993;260:810-12.

8 Bronner CE, Baker SM, Morrison PT, et al. Mutation in the DNA mismatch repair gene homologue hMLH1 is associated with hereditary non-polyposis colon cancer. Nature 1994;368:258-61.

9 Nicolaides NC, Papadopoulos N, Liu B, et al. Mutations of two PMS homologues in hereditary nonpolyposis colon cancer. Nature 1994;371:75-80.

10 Chung DC, Rustgi AK. DNA mismatch repair and cancer. Gastroenterology 1995;109:1685-99.

11 Aaltonen LA, Peltomaki P, Leach FS, et al. Clues to the pathogenesis of familial colorectal cancer. Science 1993; 260:812-16.

12 Aaltonen LA, Peltomaki P, Mecklin J-P, et al. Replication errors in benign and malignant tumours from hereditary nonpolyposis colorectal cancer patients. Cancer Res 1994; 54:1645-8.

13 Liu B, Parsons RE, Hamilton SR, et al. hMSH2 mutations in hereditary nonpolyposis colorectal cancer kindreds. Cancer Res 1994;54:4590-4.

14 Kolodner RD, Hall NR, Lipford J, et al. Structure of the human MSH2 locus and analysis of two Muir-Torr kindreds for msh2 mutations. Genomics 1994;24:516-26.

15 Liu B, Nicolaides N, Markowitz S, et al. Mismatch repair gene defects in sporadic colorectal cancers with microsatellite instability. Nat Genet 1995;9:48-55.

16 Cawkwell L, Bell SM, Lewis FA, et al. Rapid detection of allele loss in colorectal tumours using microsatellites and

17 Mecklin J, Jarvinen HJ, Hakkiluoto A, et al. Frequency of hereditary nonpolyposis colorectal cancer: a prospective hereditary nonpolyposis colorectal cancer: a prospective 588-93.

18 St John DJB, McDermott FT, Hopper JL, et al. Cancer risk in relatives of patients with common colorectal cancer. Ann in relatives of patients with $1993 ; \mathbf{1 1 8}$ :785-90.

19 Evans DGR, Walsh S, Jeacock J, et al. Incidence of hereditary nonpolyposis colorectal cancer in a population based study of 1137 consecutive cases of colorectal cancer. Br F Surg 1997;84:1281-5

20 Lothe RA, Peltomaki P, Meking GI, et al. Genomic instability in colorectal cancer: relationship to clinicopathological
variables and family history. Cancer Res 1993;53:5849-52.

1 Samowitz W, Slattery M, Kerber R. Microsatellite instability in human colonic cancer is not a useful indicator of familial colorectal cancer. Gastroenterology 1995;109:1765-71.

22 Lindblom A, Tannergard P, Werelius B, et al. Genetic mapping of a second locus predisposing to hereditary pon-polyposis colon cancer. Nat Genet 1993;5:279-82.

23 Liu B, Parsons R, Papadopoulos N, et al. Analysis of mismatch repair genes in hereditary non-polyposis colorecmismatch repair genes in hereditary non-polyp

24 Shibata D, Peinado MA, Ionov Y, et al. Genomic instability in repeated sequences is an early somatic event in colorectal tumorigenesis that persists after transformation. Nat Genet 1994;6:273-81.

25 Litt M. PCR of TG microsatellites. In: McPhearson MJ, Quirke P, Taylor GR, eds. PCR: a practical approach. New York: Oxford University Press, 1991:85-99.

26 Jass JR, Cottier DS, Jeevaratnam P, et al. Diagnostic use of microsatellite instability in hereditary non-polyposis colorectal cancer. Lancet 1995;346:1200-1.

27 Wijnen J, Meera Khan P, Vasen $\mathrm{H}$, et al. Hereditary nonpolyposis colorectal cancer families not complying with the Amsterdam criteria show extremely low frequency of mismatch repair gene mutations. Am f Hum Genet 1997;61:329-35. 